\title{
Estimation of spectrum requirements for mobile networks with self-similar traffic, handover, and frequency reuse
}

\author{
Won Seok Yang ${ }^{\mathrm{a}}$, Eun Saem Yang ${ }^{\mathrm{b}, *}$, Hwa J. Kim ${ }^{\mathrm{c}}$ and Dae K. Kim ${ }^{\mathrm{d}}$ \\ ${ }^{a}$ Department of Business Administration, Hannam University, Daejeon, Republic of Korea \\ ${ }^{\mathrm{b}}$ Department of Computer Engineering, Hallym University, Chuncheon, KangwonDo, Republic of \\ Korea \\ ${ }^{\mathrm{c}}$ Department of Electronics and Computer Engineering, Kangwon National University, Chuncheon, \\ KangwonDo, Republic of Korea \\ ${ }^{\mathrm{d}}$ Department of Information Communication and Computer Networks, Hallym College, Chuncheon, \\ KangwonDo, Republic of Korea
}

\begin{abstract}
This paper considers self-similarity in data traffic, handover, and frequency reuse to estimate the spectrum requirements of mobile networks. An approximate average cell capacity subject to a delay requirement and self-similar traffic is presented. It is shown that handover traffic can be an additional load. Spectrum requirements are calculated based on carrier demand instead of spectral efficiency, as at least one carrier is necessary to transmit even 1 bit. The cell-split operation is considered under frequency reuse of one. Estimation methods are presented using cell traffic in two cases. First, a procedure is presented that estimates cell traffic from previous networks. Second, cell traffic is assumed to follow probability distributions. Numerical examples demonstrate the impact of self-similarity, handover, and the proportion of cell-split occurrences on the spectrum requirements.
\end{abstract}

Keywords: Spectrum requirement, self-similarity, handover, frequency reuse, data traffic, QoS

\section{Introduction}

Self-similarity has been reported as a characteristic of data traffic. Examples include Ethernet, Web, FTP, telnet, and VBR traffic [9,18,25,30]. Self-similar traffic is bursty [19]. The queue length is very sensitive to the degree of self-similarity [28]. On the other hand, mobile traffic in GPRS (General Packet Radio Service) was investigated by [14]. It has been reported that mobile traffic shows self- similarity. In addition, mobility may affect performance and planning of wireless networks [1-3,13]. As a result, additional resources are required compared to Poisson traffic in mobile networks. Previous studies analyzed cell capacity using M/M/c or M/G/1 queues that assume Poisson traffic $[10,16,17,21,26]$. For example, the ITU-R model in [17] applied the Erlang $C$ formula to analyze the service channels per group for the packet communications services. In $[21,26]$, the required system capacity for packet switched

\footnotetext{
${ }^{*}$ Corresponding author. Tel.: +82 33248 2326; Fax: +82 33242 2524; E-mail: yanges@ hallym.ac.kr.
} 
services was calculated using M/G/1 non-preemptive priority queuing models. Therefore, previous studies may have underestimated the spectrum requirements when applied to self-similar data traffic.

A handover provides a continuous connection while a mobile station moves from one cell to another. A mobile station may communicate with multiple base stations (BSs) during a handover, for example during a soft handover. In this case, handover traffic can be an additional load on mobile systems. The portion of handover traffic to total traffic is approximately $12 \%$ considering only soft handovers in cdma2000 1x networks [24].

In mobile environments, each cell has a different spectrum requirement since traffic is unevenly distributed in a region. The spectrum requirement required to serve a region is not the average but the maximum demand of cells. This is the basic concept of previous methods. It is theoretically feasible but lacks the practical aspects of frequency reuse. Recent mobile technologies, including cdma2000 1x, WCDMA, and mobile WiMAX, support frequency reuse of one. All cells can operate on the same frequency channel, which allows splitting a cell into multiple cells to share the coverage and traffic of the original cell. As a result, we can reduce the spectrum requirements by using a cell-split. Not considering frequency reuse may result in the overestimation of the spectrum requirements. On the other hand, even though users at the cell edge may suffer degradation in connection quality due to heavy cochannel interference in the cell-split, the possibility of a cell-split with a frequency reuse of one is theoretically clear [7,11,12]. In addition, cell-splits are widespread in CDMA systems, which support a frequency reuse of one. For example, a mobile operator in Korea operated around 4,000 cdma2000 1x base stations in 2003, and that number increased to around 6,000 in 2009. The operator's network covered the whole country, even as far back 2000, implying that around 2000 cells are split.

Previous research has paid no attention to self-similarity, handover, and frequency reuse, although these factors affect spectrum requirements significantly. However, a recent study did consider these three factors [5]. The method used in [5], however was simple and intuitive. It considered self-similarity but merely used a parameter to reflect self-similarity without analyzing it mathematically. The scheme of the cell-split was not specific. In addition, the study did not consider when the data of the cell traffic is insufficient.

This paper presents a robust method that estimates the spectrum requirements of mobile networks considering self-similarity, handover, and a frequency reuse of one. First, we analyze an approximate average cell capacity per carrier subject to self-similar traffic considering delay for the QoS requirement, similar to $[20,23]$. A queue length with the Fractional Brownian Motion (FBM) process is utilized to obtain the mean delay. The FBM process, a generalization of the Brownian motion process, is frequently used in the analysis of self-similar traffic [28]. Second, we show that handover traffic can be an additional load in some handover technologies. Third, we present a specific procedure for estimating the spectrum requirements based on a cell-split with a frequency reuse of one. We consider two cases according to the degree of cell traffic information. First, we deal with an estimation method assuming the cell traffic is known or can be estimated from the traffic information of previous networks. Second, we extend this method to a case when the cell traffic is distributed uniformly, exponentially, and normally. Unlike previous research, the carrier bandwidth is used instead of spectral efficiency as mobile networks require at least one carrier, even when they transmit 1 bit.

The rest of this paper is organized as follows. In Section 2, the model is described. In Section 3, the average cell capacity is presented. In Section 4, handover is investigated. In Sections 5 and 6, specific estimation methods are presented. Section 7 shows numerical examples. Section 8 concludes this study. 


\section{Model description}

It is assumed that there are $K$ types of packets according to their length. Let $x_{k}$ and $p_{k}$ denote the length of a type $k$ packet and the probability that a packet is type $k$, respectively. Let $C_{F}$ denote the full capacity per carrier, for example the average throughput. Let $\lambda_{k}, s_{k}$, and $\rho_{k}$ denote the arrival rate, transmission time, and utilization of type $k$ packets, $k=1, \cdots, K$, respectively. Let $d_{k}$ denote the average delay of type $k$ packets.

Let $C_{F}$ and $C_{Q}$ denote the capacity physically provided by a system and the capacity that supports the QoS requirement, respectively. In this paper, the QoS requirement is delay. That is, $C_{Q}$ represents the maximum traffic that a system can accommodate under the condition in which the average delay is less than a target delay. Let $\hat{\lambda}_{k}$ be the maximum traffic of the type $k$ packet that fulfills the delay requirement. Then, $\hat{\lambda}_{k}$ can be calculated numerically. $C_{Q}$ is derived from $\hat{\lambda}_{k}$ and $p_{k}$. The parameter $H$ represents the Hurst parameter or the self-similarity parameter.

Let us define the handover ratio as the portion of handover traffic to the total traffic and denote it by $h$. It is assumed that $h$ is fixed in all cells. We do not consider the impact of handover mechanisms on QoS in wireless networks $[6,8]$.

It is assumed that a mobile system supports a frequency reuse of one. Accordingly, it is possible to divide a cell into multiple cells and share the coverage and traffic. This operation is called 'cell-split'. Let $M$ be the carrier requirement that stands for the number of carriers required in a region. Let $B$ and $S$ denote the carrier bandwidth and the spectrum requirement in a region, respectively. The spectrum requirement is given by $S=B M$. Let $N_{U}$ and $T_{U}$ denote the number of subscribers in a region and traffic per user, termed user traffic, respectively. Let $N_{C}$ be the number of cells in a region. Let us define $u_{k}$ as the cell traffic density which represents the traffic portion of the $k$ th cell to the total traffic in a region, for $k=1, \cdots, N_{C}$. Note that $u_{k}$ are not only values but are also distributed uniformly, exponentially, and normally. Let $G(m)$ be the cumulative distribution defined as the proportion of cells in a region that requires $m$ or less than $m$ carriers. $G(m)$ is obtained from $u_{k}, N_{U}, T_{U}, C_{Q}$ and $h$. The carrier requirement $M$ is determined as the maximum number of carriers where $G(m)$ is less than $1-\alpha$. That is, $M=\max \{m: G(m)<1-\alpha\}$. It is assumed that the cells requiring more carriers than $M$ are split. As a result, the spectrum requirements are reduced by a cell-split.

\section{Capacity analysis with self-similarity and delay requirement}

A recent study [4] reported that common packet lengths were present in actual traffic flows, although the packet lengths could vary from 7 to 65,542 bytes. Therefore, the packet length is approximated to have a discrete distribution. Using the relationship between transmission speed and capacity results in

$$
s_{k}=\frac{x_{k}}{C_{F}}, \quad \rho_{k}=\lambda_{k} s_{k}=\frac{\lambda_{k} x_{k}}{C_{F}} .
$$

According to [28], an FBM process is used frequently in the analysis of self-similar traffic. The mean queue length, denoted by $q$, with an FBM process and constant service times is given by

$$
q=\frac{\rho^{1 / 2(1-H)}}{(1-\rho)^{H /(1-H)}},
$$


where $\rho$ denotes the utilization. The closer the Hurst parameter $H$ is to 1 , the greater the degree of self-similarity. In the case of the absence of self-similarity, $H$ is 0.5. Applying Little's Law [15] to Eq. (1) results in

$$
d_{k}=\frac{\rho_{k}^{1 / 2(1-H)}}{\lambda_{k}\left(1-\rho_{k}\right)^{H /(1-H)}},
$$

where $\rho_{k}$ represents the utilization with the type $k$ packets.

Let $\hat{\lambda}_{k}$ be the maximum traffic under the condition in which the average delay in Eq. (2) is less than the target delay. Here, $\hat{\lambda}_{k}$ can be obtained numerically from Eq. (2). This gives the average capacity per carrier with a delay requirement as follows:

$$
C_{Q}=\sum_{k=1}^{K} p_{k} \hat{\lambda}_{k} x_{k}
$$

\section{Handover traffic}

Mobile networks support handover to provide mobility to users. Assuming that a mobile station communicates with multiple BSs when it moves from one cell to another, the original BS and the neighbor BSs participating in the handover use radio resources to support the handover. In this case, the traffic generated during a handover, called handover traffic, is clearly an additional load on a mobile system.

cdma2000 1x supports hard handovers, soft handovers, and softer handovers. Among these types of handovers, a mobile station communicates with multiple BSs during soft handovers and multiple sectors during softer handovers [7]. The operation of handovers in WCDMA is similar to that in cdma2000 1x [29]. In WiMAX, a mobile station communicates with all BSs in an active set during Macro Diversity Handover (MDHO) [11]. As a result, handover traffic should be considered for soft and softer handovers in CDMA and WCDMA, and MDHO in WiMAX.

The values of $N_{U}$ and $T_{U}$ can be forecasted by a market research survey. The handover ratio can be obtained from the operational data in mobile networks. Let $T_{R}$ denote the total traffic load of a network in a region. User traffic does not contain handover traffic, yielding

$$
T_{R}(1-h)=N_{U} T_{U} .
$$

\section{Estimation of the spectrum requirements with cell traffic}

Let $T_{k}$ denote the traffic loaded on the $k$ th cell, termed the cell traffic. $T_{k}$ is assumed to be unknown while $u_{k}$ is known. Using Eq. (4) results in

$$
T_{k}=u_{k} T_{R}=\frac{u_{k} N_{U} T_{U}}{(1-h)} \text { for } k=1, \cdots, N_{C} .
$$

Let us define $f_{k}$ as the number of carriers required in the $\mathrm{k}$ th cell. For convenience, $f_{k}$ has a real value. Dividing $T_{k}$ in Eq. (5) by $C_{Q}$ in Eq. (3) yields

$$
f_{k}=\frac{T_{k}}{C_{Q}}=\frac{u_{k} N_{U} T_{U}}{C_{Q}(1-h)} \quad \text { for } \quad k=1, \cdots, N_{C}
$$


Then, $G(m)$ is derived from Eq. (6).

Adding BSs increases interference, which deteriorates the call quality and the deployment cost of BSs. These factors are dependent on the mobile environments and business structures. Thus, operators can determine the acceptable range for the portion of the cell-split in a region.

Finally, it is necessary to obtain $T_{k}$ or $u_{k}$ to calculate $f_{k}$ in Eq. (6). In existing networks, $T_{k}$ is easily collected. While deploying and planning a new network, however, $T_{k}$ must be forecasted. In contrast to $N_{U}$ and $T_{U}$ in Eq. (4), it is impossible to forecast the traffic of each individual cell by a survey, as this requires numerous samples to collect the traffic information of each cell. The cell traffic density $u_{k}$ of a new network, however, can be estimated from the traffic information of a previous network that covered the same area. A mobile operator may deploy a new network while it operates different networks. For example, CDMA or GSM operators may deploy WCDMA. The cell traffic density of the latter may be similar to that of the former, as the mobility of users does not change rapidly. Consequently, although they have different amounts of traffic, they may have a similar traffic density.

It is assumed that a previous network has $J$ cells in a region. Let us divide the region into $I$ grids. Let $g_{i}$ and $b_{j}$ denote the location of grid $i$ and cell $j$, respectively. Let $L\left(g_{i}, b_{j}\right)$ denote the distance from grid $i$ and cell $j$. It is assumed that grid $i$ is included in cell $k$ if $L\left(g_{i}, b_{k}\right)$ is the smallest among all of the cells. When $i=1, \cdots, I$ and $j=1, \cdots, J$,

$$
g_{i}^{(j)}=1 \quad \text { if } \quad L\left(g_{i}, b_{j}\right) \quad \text { is minimized at } j .
$$

Otherwise, $g_{i}^{(j)}=0$. In this case, $g_{i}^{(j)}$ in Eq. (7) indicates that grid $i$ is included in cell $j$ if $g_{i}^{(j)}=1$. Let $\Pi_{j}$ denote the number of grids included in cell $j$. Using Eq. (7) yields

$$
\Pi_{j}=\sum_{i=1}^{I} g_{i}^{(j)} .
$$

Let $\Omega_{j}$ and $\omega_{i}$ denote the traffic in cell $j$ and the estimated traffic in grid $i$, respectively. It is assumed that the grids included in cell $j$ have an equal amount of traffic. This yields

$$
\omega_{i}=\Omega_{j} / \Pi_{j} \text { for } i \text { and } j \text { such that } g_{i}^{(j)}=1 .
$$

The cells in a new network can be placed differently from their previous positions in the previous network if they use a different frequency. Let $c_{k}$ denote the location of the cell $k$ in the new network. Similar to Eq. (7), let $\hat{g}_{i}^{(k)}=1$ if $L\left(g_{i}, c_{k}\right)$ is minimized at $k$ for $i=1, \cdots, I$ and $k=1, \cdots, N_{C}$. Let $\hat{\Omega}_{k}$ denote the summation of the traffic in cells included in cell $k$. This yields

$$
\hat{\Omega}_{k}=\sum_{i=1}^{I} \sum_{k=1}^{N_{C}} \hat{g}_{i}^{(k)} \omega_{i} \text { for } k=1, \cdots, N_{C} .
$$

The cell traffic density $u_{k}$ is then estimated as follows:

$$
\mu_{k}=\hat{\Omega}_{k} / \sum_{k=1}^{N_{C}} \hat{\Omega}_{k} \text { for } k=1, \cdots, N_{C} .
$$




\section{Estimation of the spectrum requirements with cell traffic distribution}

A new operator without previous networks does not have the data of cell traffic. In this case, a method is presented here that estimates the spectrum requirements assuming a certain cell traffic distribution. An exponential and a normal distribution are considered. In addition, a case in which the traffic of each cell is equal is considered.

Let $Y_{k}$ denote an independent and identical random variable for the cell traffic in the $k$ th cell for $k=1, \cdots, N_{C}$. The expected total traffic in a region is

$$
E\left(\sum_{k=1}^{N_{c}} Y_{k}\right)=N_{c} E[Y]
$$

which is equal to $T_{R}$ in Eq. (4). This yields

$$
E[Y]=\frac{N_{U} T_{U}}{N_{C}(1-h)} .
$$

Assuming that $Y$ is exponentially distributed with parameter $\theta, E[Y]=1 / \theta$. Let $\hat{\theta}$ denote an estimator of $\theta$. Applying the method of moments in [27] yields

$$
\hat{\theta}=\frac{1}{E[Y]}=\frac{(1-h) N_{C}}{N_{U} T_{U}}
$$

Let $F$ define a random variable for the required number of carriers in a cell. This corresponds to $f_{k}$ in Eq. (6) with real values. It is clear that $F=Y / C_{Q}$. This gives

$$
G(m)=P(F<m)=P\left(Y<m C_{Q}\right)=1-e^{-\hat{\theta} m C_{Q}} .
$$

Let $S_{\exp }$ denote the spectrum requirements under the assumption of an exponential cell traffic distribution. Using Eq. (11) gives

$$
S_{\exp }=\left[-\frac{\ln (\alpha) N_{U} T_{U}}{N_{C} C_{Q}(1-h)}\right] \cdot B,
$$

where the notation $[x]$ denotes the maximum integer that is less than the real value $x$.

Next, it is assumed that the traffic in each cell is equal. Hence, $T_{k}=E[Y]$. Substituting $E[Y]$ in Eq. (9) with $T_{k}$ in Eq. (6) gives

$$
f_{k}=\frac{N_{U} T_{U}}{N_{C} C_{Q}(1-h)} \text { for } k=1, \cdots, N_{C} .
$$

Let $S_{\text {equal }}$ denote the spectrum requirements when the cell traffic is equal. Let us define $M=\left[f_{k}\right]$. From Eq. (7), it is straightforward that $G(M)=0$ and $G(M+1)=1$. This yields

$$
S_{\text {equal }}=\left[\frac{N_{U} T_{U}}{N_{C} C_{Q}(1-h)}\right] \cdot B
$$

Note that the spectrum requirement is equal to Eq. (14) when $\alpha=e^{-1}$. This implies that a case with equal cell traffic is equivalent to splitting the $e^{-1}$ portion of cells with exponentially distributed cell traffic. 
Table 1

$C_{Q}$ and carrier requirements compared with $H=0.5$

\begin{tabular}{lllllll}
\hline$H$ & 0.5 & 0.6 & 0.7 & 0.8 & 0.9 & 0.95 \\
$C_{Q}$ & 8.7 & 8.5 & 8.0 & 7.0 & 5.3 & 4.4 \\
Carrier requirements & 1.0 & 1.0 & 1.1 & 1.2 & 1.7 & 2.0 \\
compared to $H=0.5$ & & & & & & \\
\hline
\end{tabular}

Table 2

$f_{k}$, an example

\begin{tabular}{ccccccccccc}
\hline$k$ & 1 & 2 & 3 & 4 & 5 & 6 & 7 & 8 & 9 & 10 \\
$f_{k}$ & 2.1 & 3.3 & 4.1 & 5.2 & 2.3 & 4.4 & 5.5 & 4.6 & 5.1 & 4.8 \\
\hline
\end{tabular}

Table 3

$G(m)$, an example

\begin{tabular}{ccccccc}
\hline$m$ & 1 & 2 & 3 & 4 & 5 & 6 \\
$G(m)$ & $0 \%$ & $0 \%$ & $20 \%$ & $30 \%$ & $70 \%$ & $100 \%$ \\
\hline
\end{tabular}

Finally, a heuristic method is presented that estimates the spectrum requirements when the cell traffic is normally distributed. Let $\hat{\mu}$ and $\hat{\sigma}^{2}$ denote the estimators of the mean and variance, respectively. Using the method of moments [27], $\hat{\mu}$ is equivalent to $E[Y]$ in Eq. (9). The random variable $Y$ is positive as it represents traffic. However, it can have a negative value because it follows a normal distribution. Let $\beta=P(Y<0)$. $\beta$ can be interpreted as a statistic pertaining to the validity of $Y$. Thus, $\hat{\sigma}^{2}$ is obtained under a given value of $\beta$.

Let $S_{\text {nor }}$ and $M_{\text {nor }}$ denote the spectrum requirements and the carrier requirement with a normal cell traffic distribution. Summarizing the above results yields

$$
S_{\text {nor }}=M_{\text {nor }} B \text {, }
$$

where $M_{\text {nor }}$ is obtained by

$$
M_{\text {nor }}=\max \left\{m: P\left(Y<m C_{Q}\right)<1-\alpha\right\},
$$

and $Y$ is normally distributed with $\hat{\mu}$ in Eq. (12) and $\hat{\sigma}^{2}$, which satisfies $P(Y<0)=\beta$.

\section{Numerical example}

Modifying the frequency of the common packet sizes as in [22] yields a discrete distribution for packet length, as follows: There are five types of packet lengths, 40, 52, 576, 1420, and 1500 bytes, with probabilities of $0.42,0.06,0.24,0.06$, and 0.22 , respectively. It is assumed that the delay requirement is 0.1 and $C_{F}=8.8 \mathrm{Mbps}$.

Table 1 shows that the case with $H=0.95$ requires double the carries of the case with $H=0.5$. This implies that more carriers are needed with higher self-similarity. Next, let us assume that there are 10 cells and $f_{k}$ in Eq. (6) has the following values. Then, we have $G(m)$ in Eq. (7) as follows. When $\alpha=$ 0.1 , the carrier requirement becomes 5 . In Table 2, three cells requiring six carriers are spilt.

Finally, we show numerical examples with a cell traffic distribution. Suppose that $B=10 \mathrm{MHz}, h=$ $12 \%, N_{C}=30, N_{U}=50000, T_{U}=23 \mathrm{Kbps}$, and $\beta=0.1 \%$. An operator can obtain the real values of the above parameters. The delay requirement depends on the QoS policy. The mobile networks that an operator deploys determines $C_{F}$ and $B$. The handover scheme that an operator implements determines 


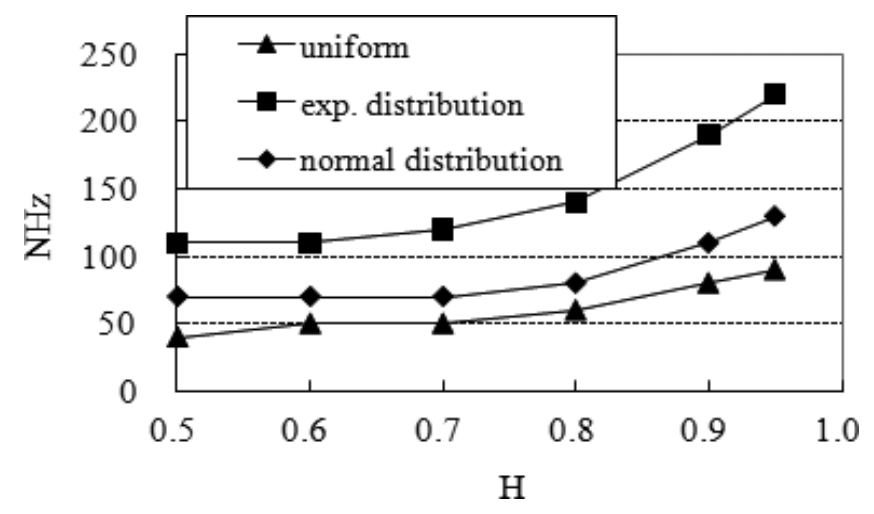

Fig. 1. Spectrum requirements over $H$ when $\alpha=10 \%$.

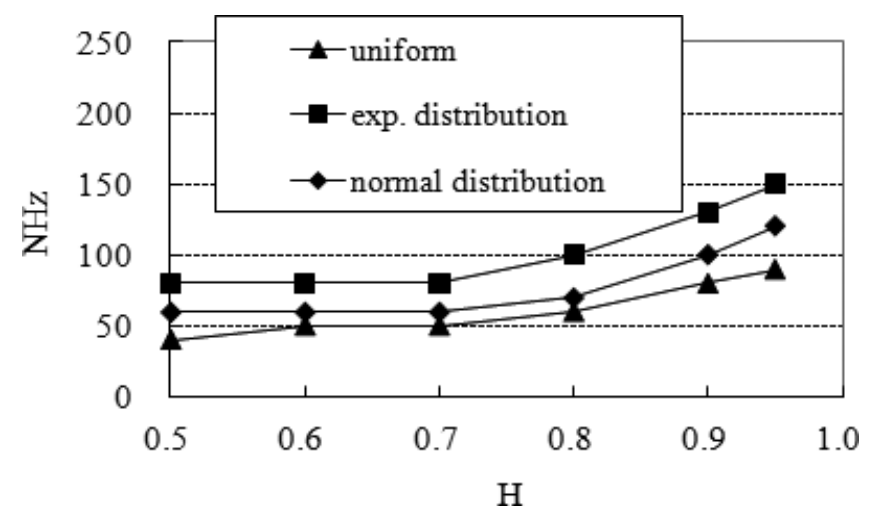

Fig. 2. Spectrum requirements over $H$ when $\alpha=20 \%$.

$h$. A mobile operator's business plan contains $N_{U}, T_{U}$, and $N_{C}$ to design mobile networks and analyze the cost and revenue.

Figure 1 shows that the spectrum requirements over Hurst parameter $H$. As mentioned in Section 1 , self-similar traffic is bursty. Traffic with higher values of $H$ requires more capacity than Poisson traffic. A mobile system requires more capacity to accommodate traffic with higher self-similarity. Accordingly, the spectrum requirements increase over $H$ in Fig. 1. This trend holds even though the cell traffic distribution changes. On the other hand, the spectrum requirements with $H=0.9$ are twice what they were with $H=0.5$. Spectrum requirements are essential parameters when a government designs and allocates the spectrum, the national resource. Consequently, ignoring self-similarity may cause estimation errors and result in an ineffective and inefficient spectrum policy.

Next, the spectrum requirements drop as $\alpha$ increases from 10\% to 20\% in Figs 1 and 2, respectively. The results are apparent since more cells are split with a higher value of $\alpha$. This observation has important implications in the economic operation of spectrums for mobile operators. Adding carriers to obtain a spectrum increases the cost. Similarly, adding base stations incurs investment and operational costs. Then, operators can analyze the optimal spectrum operation, comparing the costs between adding new carriers and adding new base stations, which implies a cell-split. Figures 3 and 4 show that an additional spectrum is needed at a higher handover ratio. The results are clear since handover traffic is an additional load on mobile systems. As a result, self-similarity, handover, and frequency reuse affect the spectrum requirements considerably. 


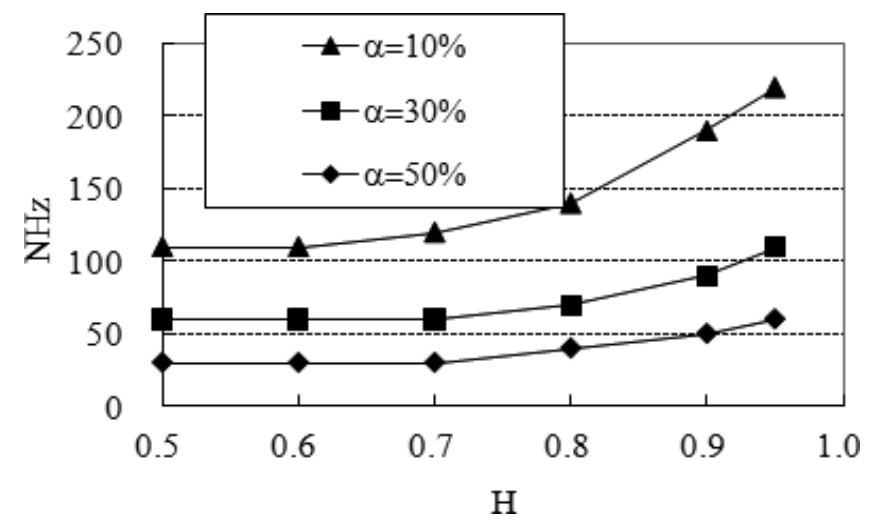

Fig. 3. Spectrum requirements over $H$ with exponential cell traffic distribution.

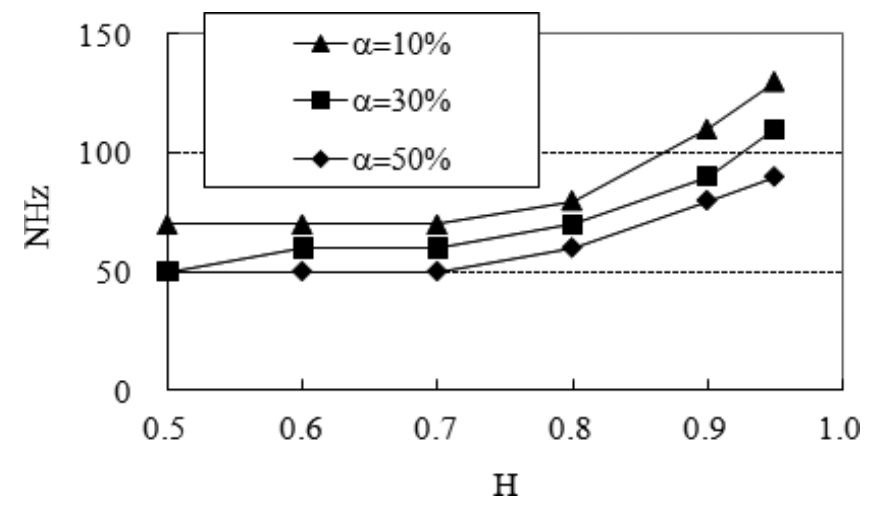

Fig. 4. Spectrum requirements over $H$ with normal cell traffic distribution.

\section{Conclusion}

This study considered three factors that should be involved in estimating spectrum requirements: selfsimilarity in data traffic, handover, and frequency reuse. An approximate average cell capacity subject to self-similar traffic was presented. It was shown that handover traffic was an additional load in some handover scenarios. The cell-split operation under the frequency reuse of one was considered. An estimation method of the spectrum requirements was presented that utilizes the cell traffic information of a previous network. In addition, the estimation method handled cell traffic distribution. Numerical examples demonstrated that the spectrum requirements were sensitive to self-similarity, handover traffic, and the proportion of cell-split operations. Our study demonstrated that considering these factors is essential to guaranteeing the accuracy of spectrum demand estimations.

\section{Acknowledgements}

This paper has been supported by the 2010 Hannam University Research Fund. This research was supported by the MKE(The Ministry of Knowledge Economy), Korea, under the ITRC(Information Technology Research Center) support program supervised by the NIPA(National IT Industry Promotion Agency) (NIPA-2010-(C1090-1011-0013)). 


\section{References}

[1] A. Doci and F. Xhafa, WIT: A wireless integrated traffic model, Mobile Information Systems 4(1) (2008), $219-235$.

[2] A. Doci, Interconnected Traffic with Real Mobility Tool for Ad Hoc Networks, ICPPW '08: Proceedings of the 2008 International Conference on Parallel Processing - Workshops, Washington, DC, USA, 2008, pp. 204-211.

[3] A. Doci, L. Barolli and F. Xhafa, Recent Advances on the Simulation Models for Ad Hoc Networks: Real Traffic and Mobility Models, Scalable Computing: Practice and Experience Scientific International Journal for Parallel and Distributed Computing 10(1) (2009), 1-11.

[4] A. Kos, M. Pustišek and J. Bešter, Characteristics of real packet traffic captured at different network locations, Procs EUROCON, Ljubljana, Slovenia, 2003.

[5] E.S. Yang, H.J. Kim and W.S. Yang, A new methodology for estimating spectrum requirements with data traffic, Proc WINSYS, Barcelona, Spain, 2007, pp. 269-272.

[6] G. Mino, L. Barolli, F. Xhafa, A. Durresi and A. Koyama, Implementation and performance evaluation of two fuzzy-based handover systems for wireless cellular networks, Mobile Information Systems 5(4) (2009), 339-361.

[7] K.I. Kim, Handbook of CDMA system design, engineering, and optimization, Prentice Hall, Upper Saddle River, NJ 07458, 2000.

[8] L. Barolli, A speed-aware handover system for wireless cellular networks based on fuzzy logic, Mobile Information Systems 4(1) (2008), 1-12.

[9] M.E. Crovella and A. Bestavros, Self-similarity in World Wide Web traffic: Evidence and possible causes, IEEE/ACM Trans Networking 5(6) (1997), 835-846.

[10] M. Matinmikko, T. Irnich and J. Huschke, A. Lappeteläinen and J. Ojala, WINNER Methodology for calculating the spectrum requirements for systems beyond IMT-2000, Proc 14th IST Mobile \& Wireless Comm. Summit, Dresden, Germany, June 2005.

[11] Mobile WiMAX - Part I: A technical overview and performance evaluation, WiMAX Forum, August 2006.

[12] Mobile WiMAX - Part II: A comparative analysis, WiMAX Forum, May 2006.

[13] P. Fülöp, S. Imre, S. Szabó and T. Szálka, Accurate mobility modeling and location prediction based on pattern analysis of handover series in mobile networks, Mobile Information Systems 5(3) (2009), 255-289.

[14] R. Kalden and S. Ibrahim, Searching for Self-Similarity in GPRS, Springer-Verlag Berlin Heidelberg, 2004, pp. 83-92.

[15] R.W. Wolff, Stochastic modeling and the theory of queues, Prentice Hall, 1989.

[16] Recommendation ITU-R M.1390, Methodology for the calculation of IMT-2000 terrestrial spectrum requirement, ITU, 1999.

[17] Report ITU-R M.2023, Spectrum requirements for international mobile telecommunications-2000 (IMT-2000), ITU, 2000.

[18] S. Dill, D. Mumar, K. MaCurley, S. Rajagopalan, D. Divakumar and A. Tomkins, Self-similarity in the Web, Proc 27th VLDB Conference, Roma, Italy, 2001.

[19] S. Kasahara, Internet traffic modeling: Markovian Approach to self-similar traffic and prediction of loss probability of finite queues, IEICE Trans Commun E84-B(8) (2001), 2134-2141.

[20] S. Parkvall and D. Astely, The Evolution of LTE towards IMT-Advanced, Journal of Communications 4(3) (2009), $146-154$.

[21] T. Irnich and B. Walke, Spectrum estimation methodology for next generation wireless systems: Introduction and results of application to IMT-2000, Proc. PIMRC 2004, Barcelona, Spain, 2004.

[22] The Applied Research Group, IP data analysis, August 9th, 2000. (http://ipmon.sprint.com).

[23] TR 36.913 V9.0.0, Requirements for further advancements for Evolved Universal Terrestrial Radio Access (E-UTRA) (LTE-Advanced) (Release 9), 3GPP, 2009.

[24] V. K Grag, IS-95 CDMA and cdma2000: cellular/PCS systems implementation, Prentice Hall PTR, Upper Saddle River, NJ 07458, 2000.

[25] W.E. Leland, M. Taqqu, W. Willinger and D.V. Wilson, On the self-similar nature of Ethernet traffic (extended version), IEEE/ACM Trans. Networking 2(1) (1994), 1-13.

[26] W.G. Chung, E. Lim, J.G. Yook and H.K. Park, Calculation of spectral efficiency for estimating spectrum requirements of IMT-Advanced in Korean mobile communication environments, ETRI Journal 29(2) (2007), 153-161.

[27] W. Mendenhall, R.L. Scheaffer and D.D. Wackerly, Mathematical statistics with applications, Third Edition, Duxbury Press, Boston, 1986.

[28] W. Stallings, High-speed networks; TCP/IP and ATM design principles, Prentice Hall, 1998.

[29] Z. Becvar, J. Zelenka and R. Bestak, Comparison of Handovers in UMTS and WiMAX, Elektro2006, Zilina 2006, ISBN:80-8070-544-5.

[30] Z. Sahinoglu and S. Tekinay, Self-similar traffic and network performance, IEEE Commun Magazine 37 (1999), 48-52. 
Won Seok Yang received his master's degree and Ph.D. from KAIST(Korea Advanced Institute of Science and Technology), Daejeon, Korea. Currently, he is a professor of Department of Business Administration at Hannam University. His research interests include stochastic modeling, queueing theory, production management, telecommunication networks and policy, information security.

Eun Saem Yang received her master's degree and Ph.D. from Kangwon National University, Chuncheon, Korea. She is currently working as a professor of Department of Computer Engineering at Hallym University. Her research interests include mobile and wireless systems, interworking of heterogeneous wired and wireless networks and mobility management.

Hwa J. Kim was born in Seoul in 1959. He received the M.S. and Ph.D. degree at KAIST(Korea Advanced Institute of Science and Technology) all in Electronics Engineering. He is currently working as a professor at Kangwon National University, Korea since 1988. His work is related to the area of communication protocol and network programming.

Dae K. Kim received his master's degree and Ph.D. from Kangwon National University, Chuncheon, Korea. Currently, He is a professor of Department of Information Communication and Computer Networks at Hallym College. His research interests include communication systems and network architecture. 

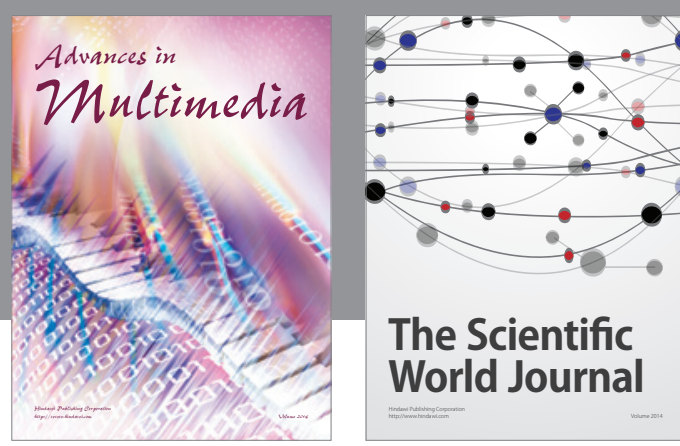

The Scientific World Journal
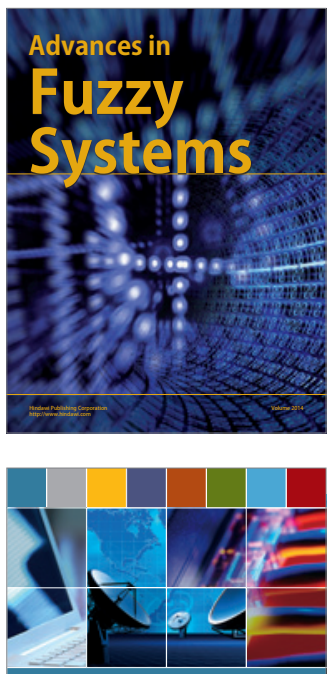

Computer Networks and Communications
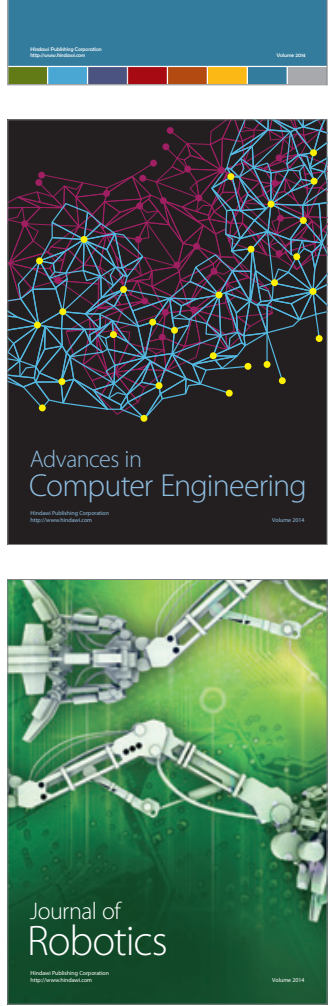
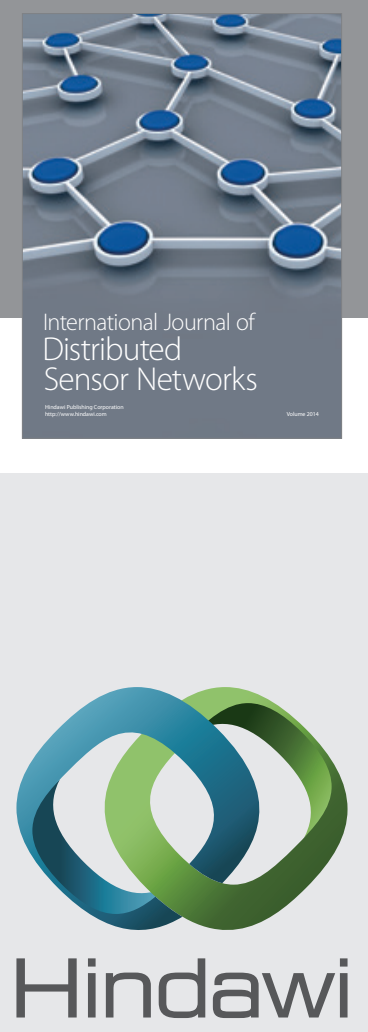

Submit your manuscripts at

http://www.hindawi.com
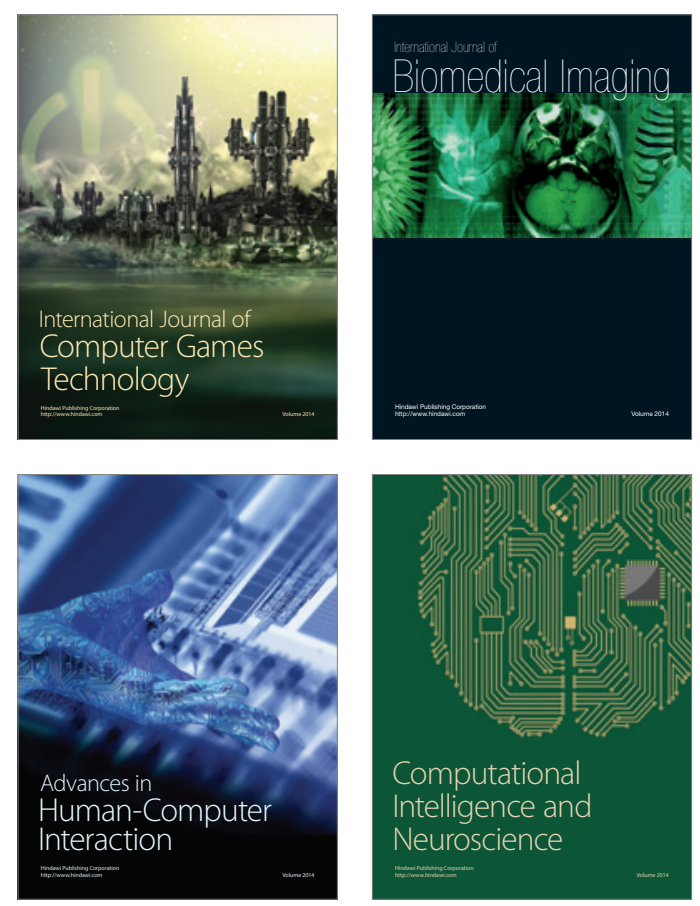
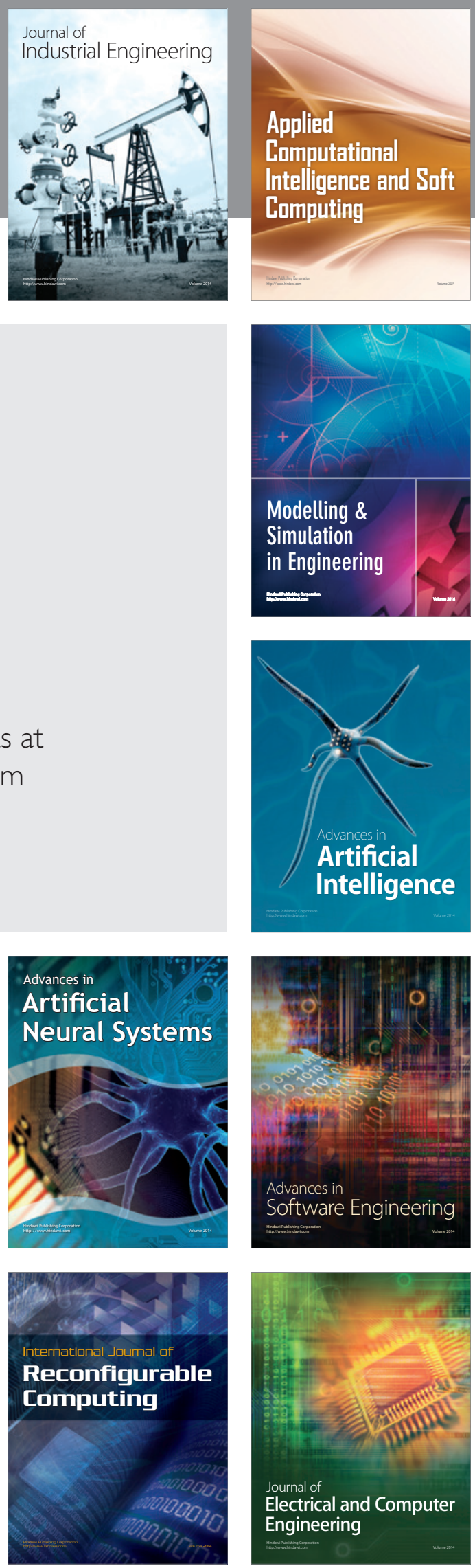